University of Nebraska - Lincoln

DigitalCommons@University of Nebraska - Lincoln

April 1999

\title{
Phase control of x-ray-atom scattering in the presence of a bichromatic laser field
}

Dejan B. Miloševíc

University of Sarajevo, Bosnia and Herzegovina

Anthony F. Starace

University of Nebraska-Lincoln, astarace1@unl.edu

Follow this and additional works at: https://digitalcommons.unl.edu/physicsstarace

Part of the Physics Commons

Miloševíc, Dejan B. and Starace, Anthony F., "Phase control of x-ray-atom scattering in the presence of a bichromatic laser field" (1999). Anthony F. Starace Publications. 161.

https://digitalcommons.unl.edu/physicsstarace/161

This Article is brought to you for free and open access by the Research Papers in Physics and Astronomy at DigitalCommons@University of Nebraska - Lincoln. It has been accepted for inclusion in Anthony F. Starace Publications by an authorized administrator of DigitalCommons@University of Nebraska - Lincoln. 


\title{
Phase control of x-ray-atom scattering in the presence of a bichromatic laser field
}

\author{
Dejan B. Miloševíc* and Anthony F. Starace \\ Department of Physics and Astronomy, University of Nebraska-Lincoln, \\ 116 Brace Laboratory, Lincoln, NE 68588-0111, USA \\ * On leave from Faculty of Science and Mathematics, Department of Physics, University \\ of Sarajevo, Zmaja od Bosne 35, 71000 Sarajevo, Bosnia and Herzegovina
}

\begin{abstract}
We consider the scattering of $50 \mathrm{eV}$ x-rays by hydrogen atoms in the presence of a bichromatic, linearly polarized laser field with frequencies $\omega$ and $r \omega$, where $r=2,3, \omega=1.17 \mathrm{eV}$, and with relative phase $\phi$ between the bichromatic laser field components. Numerical results for the differential cross-section (DCS) as a function of the number $n$, where $n \omega$ is the energy exchanged with the laser field, are presented. For either a monochromatic laser field or a bichromatic laser field with the frequencies $\omega$ and $3 \omega$, the integer $n$ can only be even, while for a bichromatic laser field with the frequencies $\omega$ and $2 \omega$, the integer $n$ can have any value. For small values of $|n|$ we find pronounced maxima in the DCS. For larger $|n|$ we find plateaus which are different for negative and positive values of $n$. For lower values of the laser field intensity the plateau for negative values of $n$ is much more extended and greater in magnitude than that for positive values of $n$. The height of either plateau is also higher for the bichromatic laser field than for either monochromatic field. In the $(\omega, 2 \omega)$ case we find the symmetry DCS $(\phi+\pi)=$ DCS $(\phi)$. We show that the relative phase $\phi$ influences the DCS so that phase control of the $x$-ray-atom scattering process is possible. For some values of $\phi$ the energy of the outgoing $x$-ray photons can be increased. Finally, a quasi-classical explanation of the results is presented.
\end{abstract}

\section{Introduction}

Laser-field induced or assisted atomic processes have attracted both theoretical and experimental interest in recent years. For example, see the latest reports in the conference proceedings edited by Muller and Fedorov (1996) and by Lambropoulos and Walther (1997). Using strong laser fields it is possible to generate harmonic photons with an energy of almost $500 \mathrm{eV}$ (Chang et al. 1997, 1999, Spielmann et al. 1997, and Schnürer et al. 1998). Such harmonic photons are a promising source of coherent soft x-ray pulses. These pulses have already been used in laser-assisted atomic experiments (Schins et al. 1994, Glover et al. 1996). In anticipation of such coherent $x$-ray sources, $x$-ray-atom scattering in the presence of a monochromatic laser field was recently considered theoretically (Miloševíc and Ehlotzky 1998). Plateau-like structures in the differential cross-section (DCS) as a function of the number of photons exchanged with the laser field were found. Besides this, recently Miloševíc and Starace (1998) considered laser-assisted x-rayatom scattering in the presence of a static electric field. It was found that the static electric field gives rise to a substantial increase of the scattered $x$-ray energies. 
In this paper we extend the theory presented by Miloševíc and Ehlotzky (1998) to the case of $x$-ray scattering in the presence of a bichromatic laser field. It is well known that the presence of a bichromatic laser field can substantially modify a host of atomic processes including molecular reactions, multiphoton ionization, autoionization, high-order harmonic generation, and free-free transitions in a laser field (see, for example, the introduction of Miloševíc and Ehlotzky (1997) and references therein). The important parameter in such investigations is the phase difference $\phi$ between the laser field components. It is generally found that the probability of an atomic or molecular process can be increased or decreased as $\phi$ is varied. This effect has been labeled "coherent phase control." We examine in this paper the possibility of coherent phase control of x-ray-atom scattering processes.

In Section 2 we present briefly the main expressions for the DCS for laser-assisted $x$-ray-atom scattering. Because a detailed derivation including discussion of the approximations used was presented by Miloševíc and Ehlotzky (1998), we concentrate here on the modifications introduced by the second laser field component. In Sections 3 and 4 we present our numerical results for a bichromatic laser field of frequencies $(\omega, 3 \omega)$ and $(\omega, 2 \omega)$, respectively. In Section 5 we present a quasi-classical explanation of some features of the results obtained. Our conclusions are given in Section 6.

\section{Theory}

The DCS for laser-assisted $x$-ray-atom scattering with absorption $(n>0)$ or emission $(n<0)$ of an energy $n \omega$ from the laser field having fundamental frequency $\omega$ is (Miloševíc and Ehlotzky 1998; in this paper we use SI units plus atomic units $(e=\bar{h}=m=1))$ :

$$
\frac{\mathrm{d} \sigma(n)}{\mathrm{d} \Omega_{\hat{K}^{\prime}}}=c^{-4} \omega_{K} \omega_{K^{\prime}}^{3}\left|T_{K, K^{\prime}}^{(+)}(n)+T_{K^{\prime}, K}^{(-)}(n)\right|^{2} .
$$

Here $\omega_{K}$ and $\omega_{K^{\prime}}=\omega_{K}+n \omega$ are the energies of the incident and scattered x-ray photons, respectively. The T-matrix elements

$$
T_{K, K^{\prime}}^{( \pm)}(n)=\int_{0}^{2 \pi} \frac{\mathrm{d} \varphi}{2 \pi} \mathcal{T}_{K, K^{\prime}}^{( \pm)}(\varphi) \exp (\mathrm{in} \varphi)
$$

are the Fourier components of the matrix elements

$$
\begin{aligned}
\mathcal{T}_{\boldsymbol{K}, \boldsymbol{K}^{\prime}}^{( \pm)}(\varphi)= & \int_{0}^{\infty} \mathrm{d} \tau \int \mathrm{d} \boldsymbol{q}\left\langle\psi_{0}\left|\boldsymbol{r} \cdot \hat{e}_{\boldsymbol{K}}\right| \boldsymbol{q}+\boldsymbol{A}(t)\right\rangle \\
& \times\left\langle\boldsymbol{q}+\boldsymbol{A}(t-\tau)\left|\boldsymbol{r} \cdot \hat{e}_{\boldsymbol{K}^{\prime}}\right| \psi_{0}\right\rangle \exp \left\{-\mathrm{i}\left[S(\boldsymbol{q} ; t, \tau) \pm \omega_{\boldsymbol{K}^{\prime}} \tau\right]\right\}
\end{aligned}
$$

where $\varphi \equiv \omega t$. In Equation (3), $\left|\psi_{0}\right\rangle$ is the atomic ground state ket, $|\boldsymbol{q}\rangle$ is the plane wave ket for the electron, and $\hat{e}_{K}$ and $\hat{e}_{K^{\prime}}$ are the unit polarization vectors of the incident and scattered photon, respectively. $A(t)$ is the vector potential, defined below, and $S(q ; t, \tau)$ is the quasi-classical action for the electron in the presence of a bichromatic laser field, $S(q ; t, \tau)=\int_{t-\tau}^{t} \mathrm{~d} t^{\prime}\left\{1 / 2\left[q+A\left(t^{\prime}\right)\right]^{2}+I_{0}\right\}$, where $I_{0}$ is the atomic ionization potential. In this paper we consider only the case of the hydrogen atom, for which $I_{0}=0.5 \mathrm{au}$. The matrix element $T_{K^{\prime}, K^{(-)}}^{(n)}$ corresponds to the processes in which an x-ray photon having wavevector $K$ and energy $\omega_{K}$ is absorbed first. The atom gets ionized and the electron propagates under the influence of the laser field during the time interval from $t-\tau$ to $t$, at which time it comes back to the atomic core (i.e., the return time is $\tau$ ). At this instant the electron recombines with the atomic core, exchanging the energy $n \omega$ with the laser field and emitting an x-ray photon having wavevector $K^{\prime}$ and energy $\omega_{K^{\prime}}=\omega_{K}+n \omega$. The matrix element $T_{K, K^{\prime}}^{(+)}(n)$ describes the process in which the x-ray photon having wavevector 
$K^{\prime}$ and energy $\omega_{K^{\prime}}$ is emitted first, followed by the electron propagation in the laser field, and, finally, the absorption of an x-ray photon having wavevector $K$ and energy $\omega_{K}$ accompanied by the absorption (or emission) of an energy $n \omega$ from the laser field. The T-matrix elements in (1) are essentially five-dimensional integrals. In Miloševíc and Ehlotzky (1998) it was shown that the three dimensional integral over the intermediate electron momenta can be carried out using the timedependent WBK approximation. The integral over the return time $\tau$ in Equation (3) is computed numerically, and, finally, the T-matrices, which appear in Equation (1), are obtained using the fast Fourier transform method. It should also be mentioned that the main contribution to the DCS comes from the matrix element $T_{K^{\prime}, K}^{(-)}(n)$. Indeed, we find that for $|n|>5$, the corrections due to the term $T_{K, K^{\prime}}^{(+)}(n)$ are less than $1 \%$. This fact considerably simplifies the calculations because $\mathcal{T}^{(-}$ ${ }_{K^{\prime}, K_{K}}(\varphi)$ does not depend on $n$, which is in contrast to the term $\mathcal{T}_{K, K^{\prime}}^{(+)}(\varphi) \sim \exp [-$ $\left.\mathrm{i}\left(\omega_{K}+n \omega\right) \tau\right]$, which obviously depends on $n$. In other words, in the case that the integrand function $f(\varphi)$ in $F(n)=(2 \pi)^{-1} \int_{0}^{2 \pi} \mathrm{d} \varphi f(\varphi) \exp (\mathrm{i} n \varphi)$ does not depend on $n$, one can obtain the whole spectrum $\mathrm{F}(n)$ by computing $f(\varphi)$ and using the fast Fourier transform method. In contrast, if $f=f(\varphi, n)$, then the integral over $\varphi$ must be computed separately for each $n$.

The explicit analytical form of the matrix elements in Equation (3) is given in Miloševíc and Ehlotzky (1998) in terms of the vector potential $A(t)$, the stationary momentum $\boldsymbol{q}_{\mathrm{s}^{\prime}}$ and the action $S_{\mathrm{s}}$. The latter two quantities are defined by

$$
\begin{aligned}
& q_{\mathrm{s}} \equiv q_{\mathrm{s}}(t, \tau)=-\frac{1}{\tau} \int_{t-\tau}^{t} \mathrm{~d} t^{\prime} \boldsymbol{A}\left(t^{\prime}\right) \\
& S_{\mathrm{s}} \equiv S\left(\boldsymbol{q}_{\mathrm{s}} ; t, \tau\right)=\int_{t-\tau}^{t} \mathrm{~d} t^{\prime}\left\{\frac{1}{2}\left[q_{\mathrm{s}}(t, \tau)+\boldsymbol{A}\left(t^{\prime}\right)\right]^{2}+I_{0}\right\} .
\end{aligned}
$$

The vector potential $A(t)$ corresponds to a linearly polarized electric field vector

$$
\boldsymbol{E}(t)=\left[E_{01} \sin \omega t+E_{0 r} \sin (r \omega t+\phi)\right] \hat{e} .
$$

In Equation (5), $\hat{e}$ is the unit polarization vector, $E_{01}$ and $E_{0 \mathrm{r}}$ are the amplitudes of the two components of the laser electric field vector, which correspond to the frequencies $\omega$ and $r \omega$, respectively, and $\phi$ is the relative phase between the bichromatic laser field components. We will consider two cases: $r=2$ and $r=3$. From Equation (5) it follows that

$$
\boldsymbol{A}(t)=-\int^{t} \boldsymbol{E}\left(t^{\prime}\right) \mathrm{d} t^{\prime}=\left[A_{01} \cos \omega t+A_{0 r} \cos (r \omega t+\phi)\right] \hat{e}
$$

and

$$
\alpha(t)=\int^{t} \mathrm{~d} t^{\prime} \boldsymbol{A}\left(t^{\prime}\right)=\left[\alpha_{01} \sin \omega t+\alpha_{0 r} \sin (r \omega t+\phi)\right] \hat{\boldsymbol{e}}
$$

where $A_{0 j}=E_{0 j} /(j \omega)$ and $\alpha_{0 j}=A_{0 j} /(j \omega), j=1, r$. We introduce also the ponderomotive potentials of the laser field components $U_{\mathrm{pj}}=A_{0 j}^{2} / 4, j=1, r$, and the ponderomotive potential of the total laser field $U_{\mathrm{p}}=U_{\mathrm{p} 1}+U_{\mathrm{pr}}$. Using equations (4) and (7), the stationary momentum can be written as $\boldsymbol{q}_{\mathrm{s}}(t, \tau)=[\alpha(t-\tau)-\alpha(t)] / \tau$, while the stationary action is given by

$$
S_{\mathrm{s}}(t, \tau)=\left(I_{0}+U_{\mathrm{p}}\right) \tau-\frac{1}{2} q_{\mathrm{s}}^{2} \tau+\mathcal{U}_{1}(\omega t)-\mathcal{U}_{1}(\omega(t-\tau))
$$

where

$$
\begin{aligned}
\mathcal{U}_{1}(\varphi)=\frac{1}{2 \omega}[ & \left.U_{\mathrm{p} 1} \sin 2 \varphi+\frac{1}{r} U_{\mathrm{p} r} \sin (2 r \varphi+2 \phi)\right] \\
& +\frac{2}{\omega}\left(U_{\mathrm{p} 1} U_{\mathrm{p} r}\right)^{1 / 2}\left[\frac{1}{r-1} \sin ((r-1) \varphi+\phi)+\frac{1}{r+1} \sin ((r+1) \varphi+\phi)\right] .
\end{aligned}
$$


In the case when $r=2$, the DCS satisfies the symmetry property $\mathrm{d} \sigma(\phi+\pi) / \mathrm{d} \Omega$ $=\mathrm{d} \sigma(\phi) / \mathrm{d} \Omega$, as can be seen from the following considerations. From equations (6), (7), and (9), for $r=2$ and $\varphi=\omega t$, it follows that $A(\varphi+\pi, \phi+\pi)=-A(\varphi, \phi), \alpha(\varphi+$ $\pi, \phi+\pi)=-\alpha(\varphi, \phi)$, and $\mathcal{U}_{1}(\varphi+\pi, \phi+\pi)=\mathcal{U}_{1}(\varphi, \phi)$. Using these results and Equation (8), we obtain that the substitution $(\varphi, \phi) \rightarrow(\varphi+\pi, \phi+\pi)$ changes the sign of the stationary momentum $\boldsymbol{q}_{\mathrm{s}}$, while the action $S_{\mathrm{s}}$ remains unchanged. Taking into account the explicit form of the matrix elements in Equation (3), which is given in Miloševíc and Ehlotzky (1998), we obtain that $\mathcal{T}_{K, K^{\prime}}^{( \pm)}(\varphi+\pi, \phi+\pi)=\mathcal{T}_{K, K^{\prime}}^{( \pm)}(\varphi, \phi)$ for $r=2$. From this we easily obtain that the DCS, Equation (1), is symmetric with respect to the substitution $\phi \rightarrow \phi+\pi$.

Let us now consider how much energy, $n \omega$, the laser may contribute. By parity conservation, the number of photons exchanged with the laser field, which we denote by $n_{\mathrm{e}^{\prime}}$ must be even. If we denote the number of photons exchanged with the $j$ th component of the laser field by $n_{j}$, then $n \omega=n_{1} \omega+n_{r} r \omega$, and $n_{\mathrm{e}}=n_{1}+n_{\mathrm{r}}=$ $2 m$, where $m$ is an arbitrary integer. For $r=3$, we have $n=n_{1}+3 n_{3}=2\left(m+n_{3}\right)$, i.e., $n$ must be even, while for $r=2$ it is $n=n_{1}+2 n_{2}=2 m+n_{2}$, which is even for $n_{2}$ even and odd for $n_{2}$ odd. Therefore, in the $(\omega, 3 \omega)$ case the number $n$ can only be even, while in the $(\omega, 2 \omega)$ case it can be either even or odd.

In Sections 3 and 4 we present our numerical results for a bichromatic laser field of frequencies $(\omega, 3 \omega)$ and $(\omega, 2 \omega)$, respectively, where $\omega=1.17 \mathrm{eV}$. The intensities of both laser field components are assumed to equal $10^{14} \mathrm{~W} \mathrm{~cm}^{-2}\left(E_{01}=E_{0 r}\right)$ and the polarization vectors of the incident and scattered $x$-ray photons are taken parallel to the polarization vector of the linearly polarized laser field. As discussed by Miloševíc and Ehlotzky (1998), the case of parallel polarization vectors maximizes one of the geometrical factors occurring in the expression for the DCS for the case of arbitrary polarizations. For simplicity, we therefore only consider this case in this paper. The energy of the incident x-ray photons is $\omega_{K}=50 \mathrm{eV}$.

For laser field intensities of order $10^{14} \mathrm{~W} \mathrm{~cm}^{-2}$ and for long laser pulses, the depletion of the $\mathrm{H}$ atoms due to ionization can be important. For example, using the formula for the tunneling ionization rate from Ilkov et al. (1992), we obtain that, after 20 optical cycles of a monochromatic Nd:YAG laser pulse having intensity $10^{14} \mathrm{~W} \mathrm{~cm}^{-2}, 87 \%$ of the atoms survive (both for the first and the second harmonic), while for the third harmonic the survival probability is $90 \%$. However, our numerical calculations show that the main contribution to the laser-assisted, $x$-ray-atom scattering DCS comes from the first few optical cycles and, therefore, we neglect the depletion effect, as was done also by Miloševíc and Piraux (1996) for the high-order harmonic generation process.

\section{Results for the $(\omega, 3 \omega)$ case}

Figure 1 shows the results for the DCS as a function of the number $n$ for different values of the relative phase: (a) $\phi=0$, (b) $\phi=\pi / 2$ and (c) $\phi=\pi$. For comparison, the results for the monochromatic laser field with $E_{0}=E_{01}$ are also presented. It should be mentioned that for some values of $n$ between the plateau and the peak around $n=0$, a higher precision of computation is necessary owing to extensive cancellations which produce a minimum in the DCS. In Miloševíc and Ehlotzky (1998), for these points the upper limit of the integral over the return time $\tau$ was limited to $7 T$ (where $T=2 \pi / \omega$ ), while the present results are obtained with an upper limit of $120 \mathrm{~T}$. We emphasize that changing the upper limit of integration from $7 T$ to $120 \mathrm{~T}$ only results in differences in the DCS within the minimum. With this higher precision, the monochromatic DCS for these points is smaller than presented in Miloševíc and Ehlotzky (1998). Taking into account that 

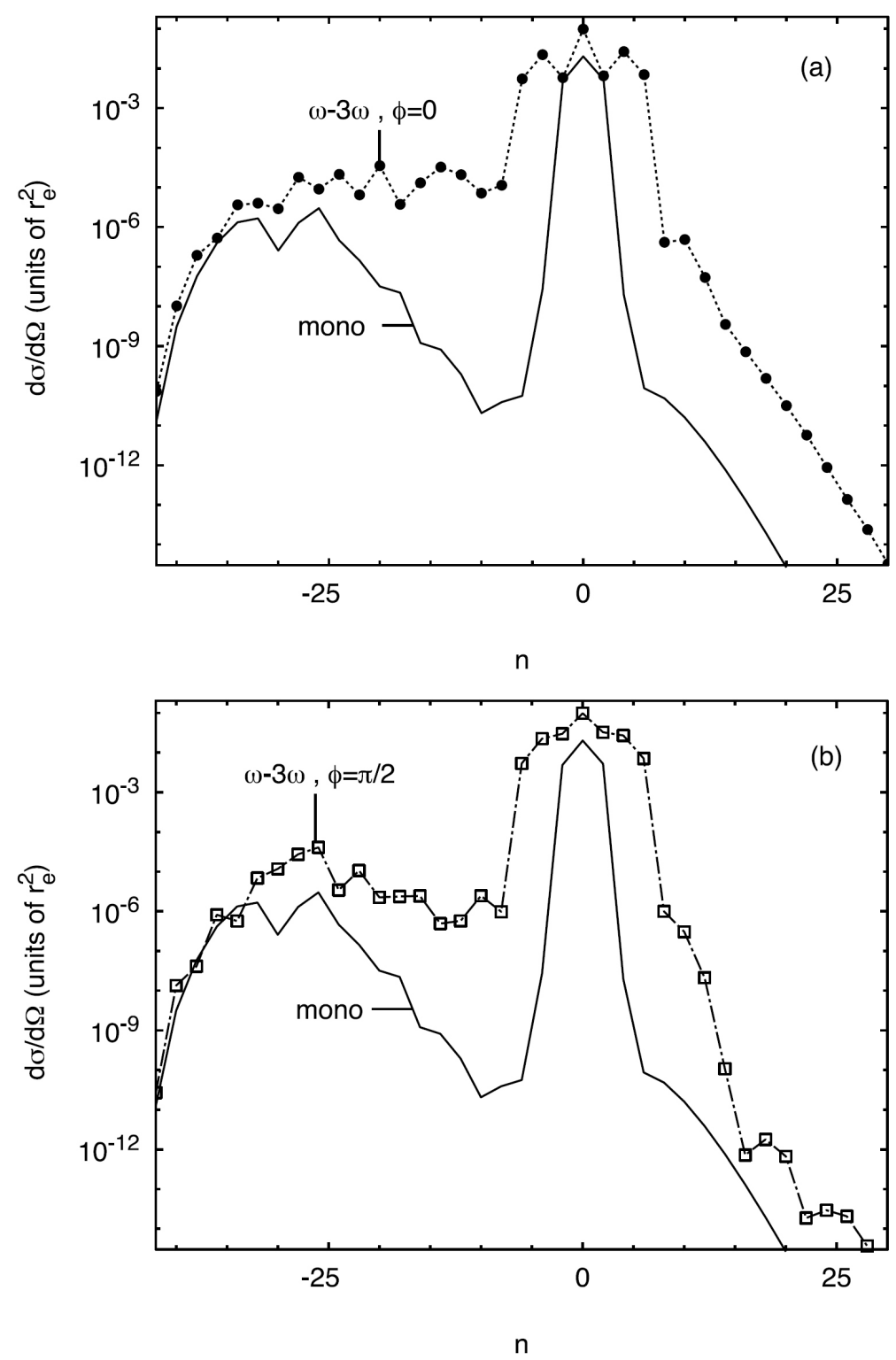

Figure 1. The DCS of x-ray-hydrogen atom scattering in units of $r_{\mathrm{e}}^{2}$ (where $r_{\mathrm{e}}=2.8 \times 10^{-15} \mathrm{~m}$ is the classical electron radius) as a function of the number $n$. $n \omega$ is the energy exchanged with either the monochromatic laser field of frequency $\omega=1.17 \mathrm{eV}$ (curve denoted by "mono") or with the bichromatic laser field with frequencies $(\omega, 3 \omega)$ and the relative phases: (a) $\phi=0$, (b) $\phi=\pi / 2$, and (c) $\phi=\pi$ (see next page). The intensities of all field components are $10^{14} \mathrm{~W} \mathrm{~cm}^{-2}$. The energy of the incident $\mathrm{x}$ ray photons is $\omega_{K}=50 \mathrm{eV}$.

for such long laser pulses the depletion of the $\mathrm{H}$ atom ground state becomes important, it may be that these minima for small $n<0$ of the monochromatic DCS are even lower. Figure 1 shows that, as in the monochromatic case, the number $n$ can only be even, but that now the DCS is increased by many orders of magnitude, depending on the values of $n$ and on the relative phase $\phi$. For example, around $n=-26$ and for $\phi=\pi$, the bichromatic DCS is two orders of magnitude 


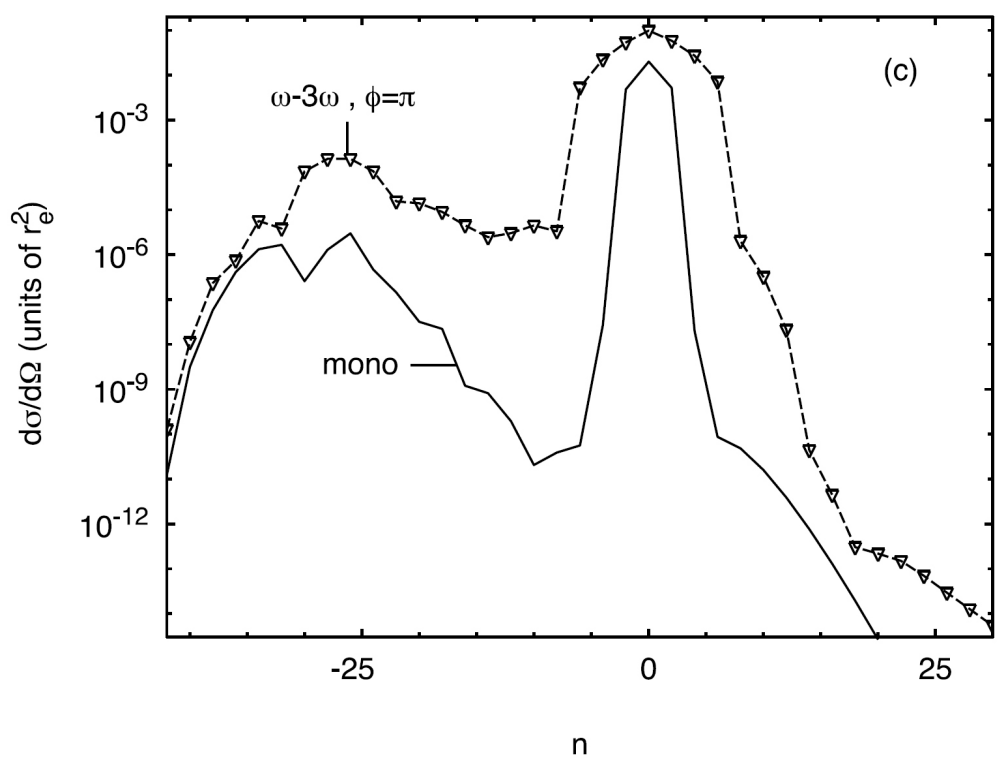

Figure 1. (continued)

larger than the monochromatic one. For lower values of $|n|$, besides the maximum for $n=0, \pm 2$, which exists also in the monochromatic case, now this maximum is broadened to include also $n= \pm 4, \pm 6$. For $|n|>2$, the DCS in the bichromatic case is much larger than in the monochromatic case and also more structure appears in the $\mathrm{d} \sigma(n) / \mathrm{d} \Omega$ curve for positive values of $n<26$.

In Figure 2 we present the bichromatic DCS as a function of the relative phase $\phi$, for three different values of $n$ : (a) $n=-26$, (b) $n=-14$, and (c) $n=12$. One can see that the DCS depends strongly on the phase $\phi$. For the cases presented, the maxima are at: (a) $\phi=3.46$, (b) $\phi=4.8$, and (c) $\phi=0$ radians. As mentioned in the introduction, these results imply that coherent phase control of the x-ray-atom scattering process is possible.

\section{Results for the $(\omega, 2 \omega)$ case}

In Figures 3 and 4, we present our results for a bichromatic laser field of frequencies $\omega$ and $2 \omega$. Because in this case the DCS satisfies the symmetry property $\mathrm{d} \sigma(\phi+\pi) / \mathrm{d} \Omega=\mathrm{d} \sigma(\phi) / \mathrm{d} \Omega$, it is enough to present the results for $\phi \in[0, \pi]$. The main new feature of the results is that now the number $n$ can be not only even, but also odd, as was explained in section 2. Similarly, as in the $(\omega, 3 \omega)$ case, the DCS is much larger in the bichromatic case than in the monochromatic one. Whereas the maximum in the monochromatic case is defined by $n=0, \pm 2$, the maximum in the bichromatic case is broader, including also $n= \pm 1 ; \pm 3$; \pm 4 . The case $n=0$ corresponds to elastic scattering in which no photons are exchanged with the laser field, $n=+1$ corresponds to the emission of one photon of frequency $\omega$ and the absorption of one photon of frequency $2 \omega$, etc. For $\phi=0$, there is a more pronounced plateau for positive values of $n$ in the range $10 \leq n \leq 15$. An explanation of the position of the cutoff of the plateau for high $n$ is given in the next section. As a function of the relative phase, for the chosen negative values of $n$ presented in Figures $4(\mathrm{a})(n=-26)$ and (b) $(n=-14)$, the DCS exhibits an oscillatory behavior. The maxima are for $\phi=0.472$ radians $(n=-26)$ and $\phi=0.98$ radians $(n=-14)$. For the chosen positive values of $n$ (Figure $4(\mathrm{c}), n=12,13$ ), the DCS has maxima for $\phi=0$ and 

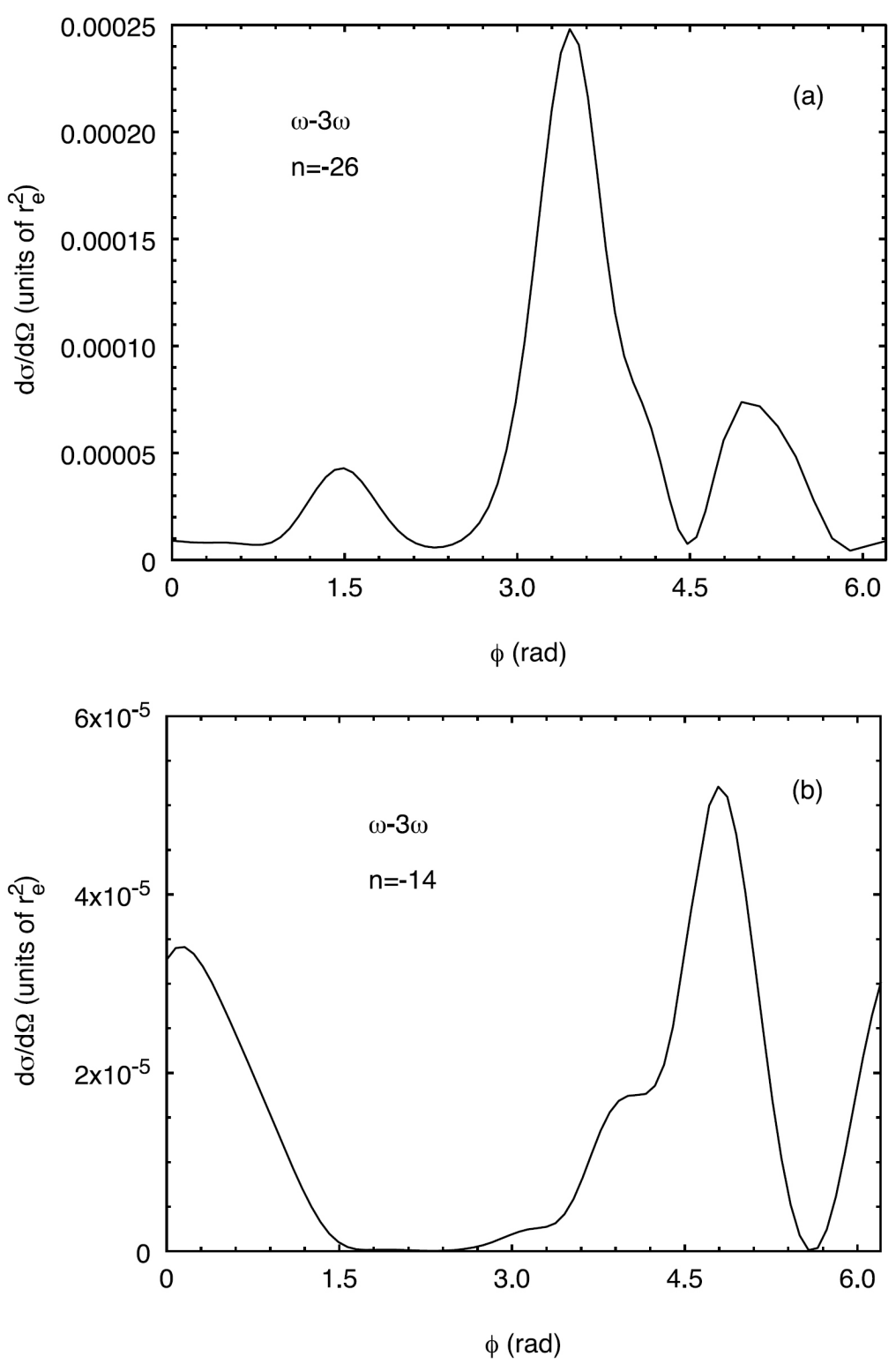

Figure 2. The DCS of x-ray-hydrogen atom scattering as a function of the relative phase $\phi$ of the same bichromatic laser field as in Figure 1. The number $n$ is: (a) $n=-26$, (b) $n=-14$, and (c) $n=12$ (see next page).

$\phi=\pi$ and a minimum for $\phi=\pi / 2$. As in the $(\omega, 3 \omega)$ case, the results presented here imply the possibility of coherent phase control of x-ray scattering by atoms.

\section{Quasi-classical analysis}

A simple quasi-classical analysis of the x-ray spectra can be carried out by applying the saddle-point method to the integral over the return time $\tau$ in Equation (3) and to the integral over the time $t$, i.e. to the integral over $\varphi=\omega t$ in the matrix element $T_{K^{\prime}, K}^{(-)}(n)$ in Equation (2) (we will consider here only this ma- 


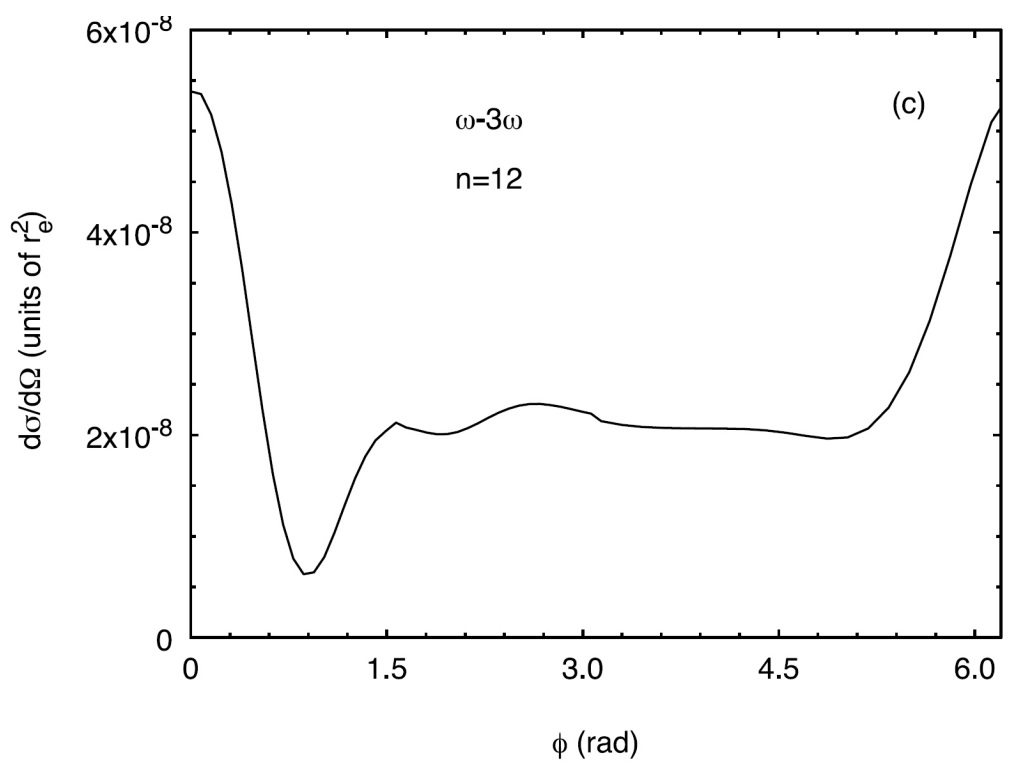

Figure 2. (continued)

trix element because it gives the main contribution to the DCS; see Miloševíc and Ehlotzky 1998). For the integral over $\tau$, the saddle-point method implies the condition $\partial\left[S(\boldsymbol{q} ; t, \tau)-\omega_{\boldsymbol{K}} \tau\right] / \partial \tau=0$, which gives

$$
\frac{1}{2}[q+\boldsymbol{A}(t-\tau)]^{2}=\omega_{K}-I_{0} .
$$

According to this equation the atom is ionized by absorbing one $x$-ray photon of energy $\omega_{K}$ and the electron is born at some time $t_{0}=t-\tau$. The left-hand side of Equation (10) is the instantaneous kinetic energy of this electron at the time $t_{0}$. In our case of a linearly polarized laser field, the initial momentum of this electron can be $p_{0}= \pm p_{0} \hat{e}$, where $p_{0}=\left[2\left(\omega_{K}-I_{0}\right)\right]^{1 / 2}$ (for other directions of $p_{0}$, the electron never returns to the atomic core, i.e. it is ionized). We are interested in the process in which, at some time $t=t_{1}$, the electron returns to the nucleus, recombines and an x-ray photon of energy $\omega_{K^{\prime}}$ is emitted. In the following we will simplify the notation by omitting the vector $\hat{e}$ and assuming that $A(t)=A(t) \hat{e}$ and $\alpha(t)=\alpha(t) \hat{e}$. From Equation (10), for $t=t_{1}, t_{1}-t_{0}=\tau$, and $\boldsymbol{q}=\boldsymbol{q}_{\mathrm{s}}=\boldsymbol{q}_{\mathrm{s}} \hat{\boldsymbol{e}}$, it follows that

$$
q_{\mathrm{s}}\left(t_{1}, \tau\right)=\frac{\alpha\left(t_{0}\right)-\alpha\left(t_{1}\right)}{t_{1}-t_{0}}=-A\left(t_{0}\right) \pm p_{0} .
$$

It is interesting that Equations (10) and (11) are equivalent to the solution of the classical Newton equation of motion for an electron in the laser field, under the condition that this electron is born at the nucleus, $r\left(t_{0}\right)=0$, with the initial momentum $p_{0}= \pm p_{0} \hat{e}$, and that at the time $t_{1}$ it returns to the nucleus, i.e. $r\left(t_{1}\right)=0$. Namely, the equation of motion of the electron in the laser field under the influence of the Lorentz force is $\ddot{r}(t)=-E(t)$. The solution of this equation is $r(t)=$ $r\left(t_{0}\right)+\alpha(t)-\alpha\left(t_{0}\right)+\left[p_{0}-A\left(t_{0}\right)\right]\left(t-t_{0}\right)$, which, under the above-mentioned conditions, is equivalent to Equations (10) and (11). A similar analysis has been performed for the high-order harmonic generation process (Lewenstein et al. 1994, Miloševíc and Piraux 1996), but, in this case, on the right-hand side of Equation (10) one has only $-I_{0}$, so that the solution of this equation over $t$ and $\tau$ exists only 

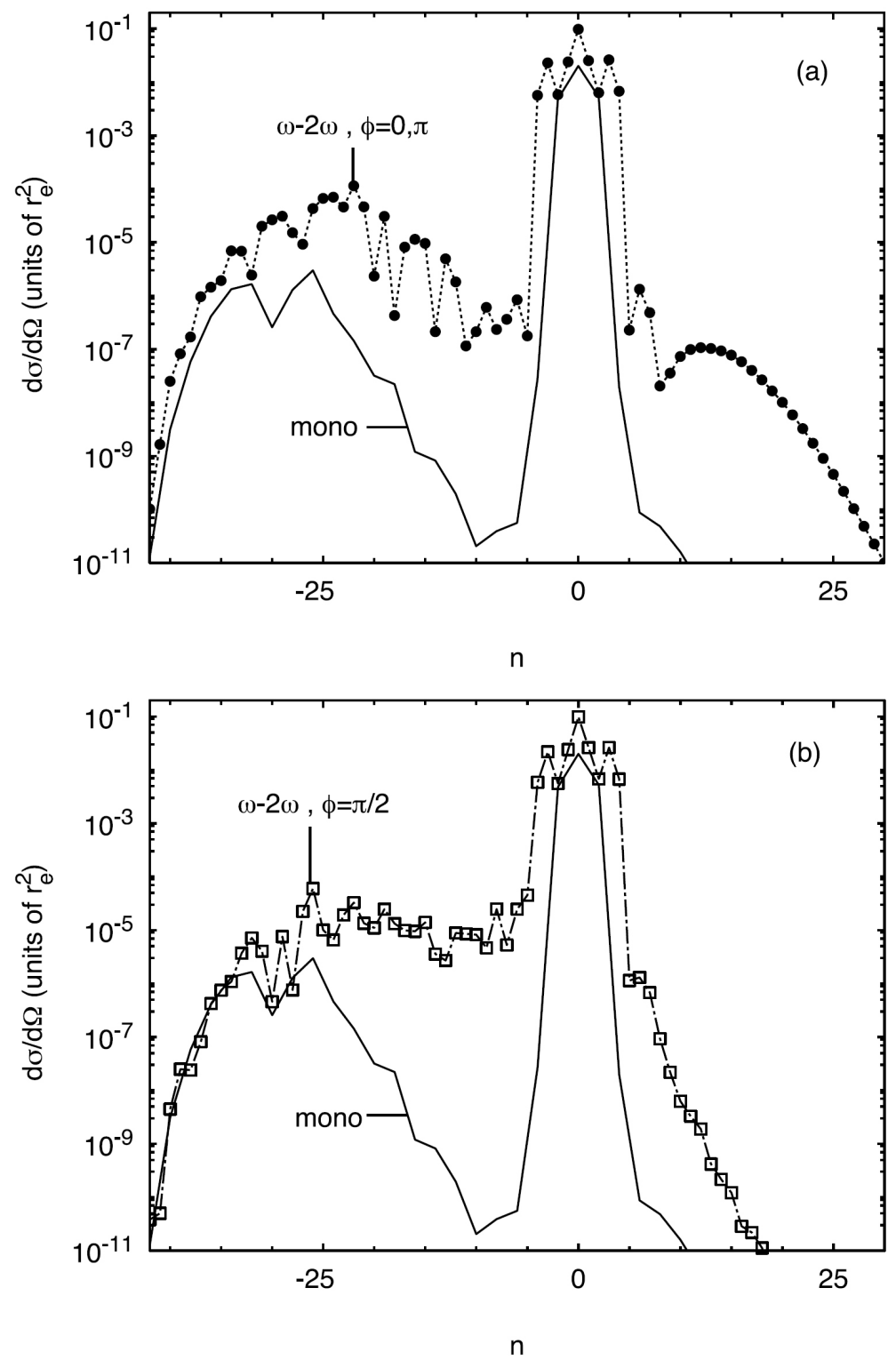

Figure 3. As in Figure 1, but for the bichromatic laser field of frequencies $(\omega, 2 \omega)$ and the relative phases: (a) $\phi=0$ and $\phi=\pi,(b) \phi=\pi / 2$.

for complex values of $t$ and $\tau$. In the limit $I_{0} \rightarrow 0$, the results of Lewenstein $e t$ al. (1994) and Miloševíc and Piraux (1996) are equivalent to the solution of the classical equation of motion for $\boldsymbol{p}_{0}=0$. In our case $\omega_{K}>I_{0}$, so that solutions for $t$ and $\tau$ are real, and there is no need for such approximation.

The application of the saddle-point method to the integral over the time $t$ implies that $\partial[S(\boldsymbol{q} ; t, \tau)-n \omega t] / \partial t=0$, so that we obtain the condition

$$
\frac{1}{2}[q+A(t)]^{2}-\frac{1}{2}[q+A(t-\tau)]^{2}=n \omega .
$$



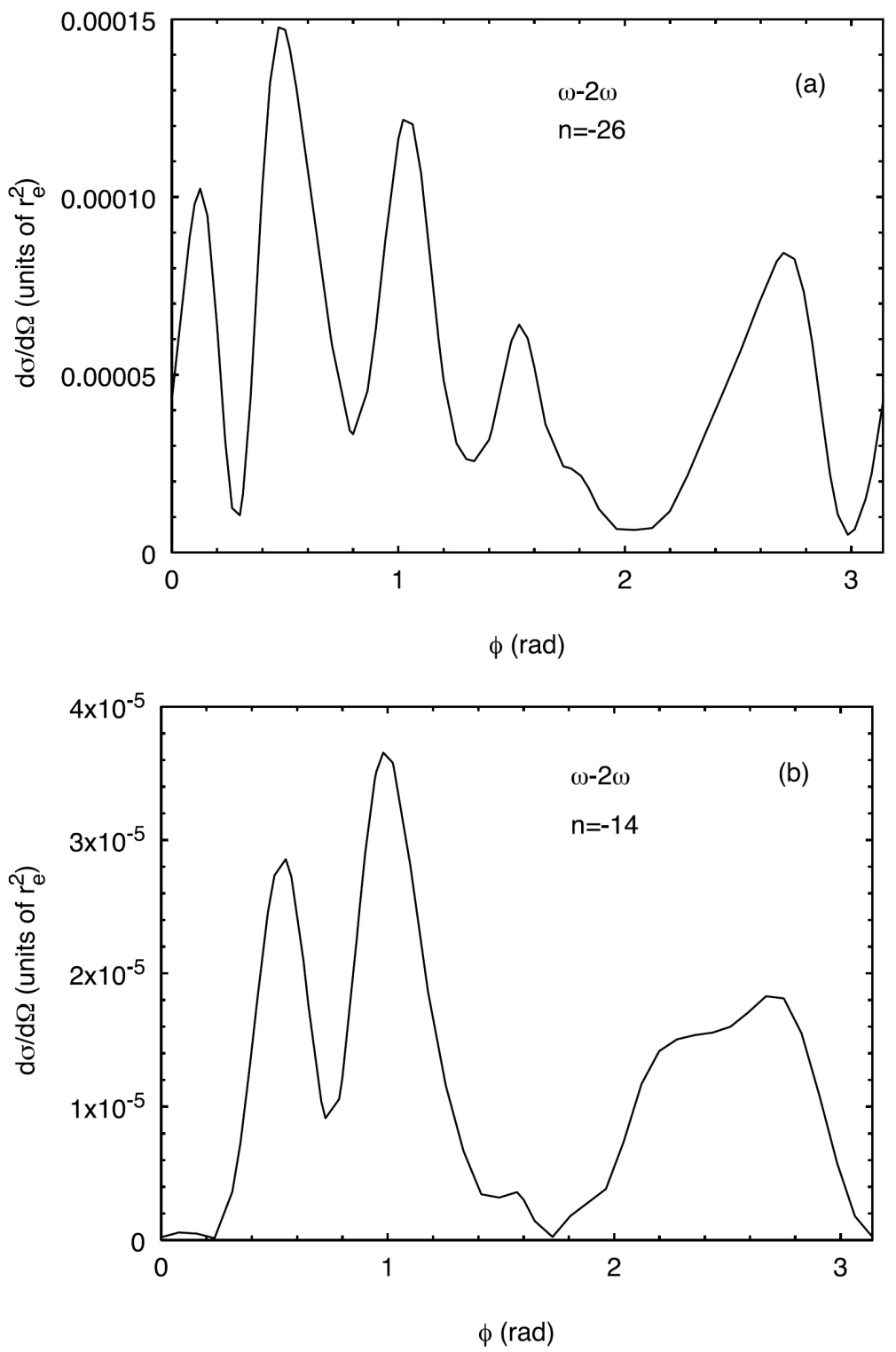

Figure 4. As in Figure 2, but for the bichromatic laser field of frequencies $(\omega, 2 \omega)$ and (a) $n=-26$, (b) $n=-14$, and (c) $n=12$ and $n=13$ (see opposite page).

Introducing (10) into (12), we obtain that the energy exchanged with the laser field at time $t_{1}$ is

$$
n \omega=\frac{1}{2}\left[\boldsymbol{q}_{\mathrm{s}}\left(t_{1}, \tau\right)+\boldsymbol{A}\left(t_{1}\right)\right]^{2}-\omega_{K}+I_{0} .
$$

We want to find the maximum (positive $n$ ) and the minimum (negative $n$ ) of this energy. We will determine them by the condition $\partial(n \omega)=\partial t_{0}=0$, i.e. by the condition that the electron is born at such time $t_{0}$ for which $n \omega$ has an extremum. Introducing (11) into (13), we obtain

$$
n \omega=\frac{1}{2}\left[A\left(t_{1}\right)-A\left(t_{0}\right) \pm p_{0}\right]^{2}-\omega_{K}+I_{0} .
$$




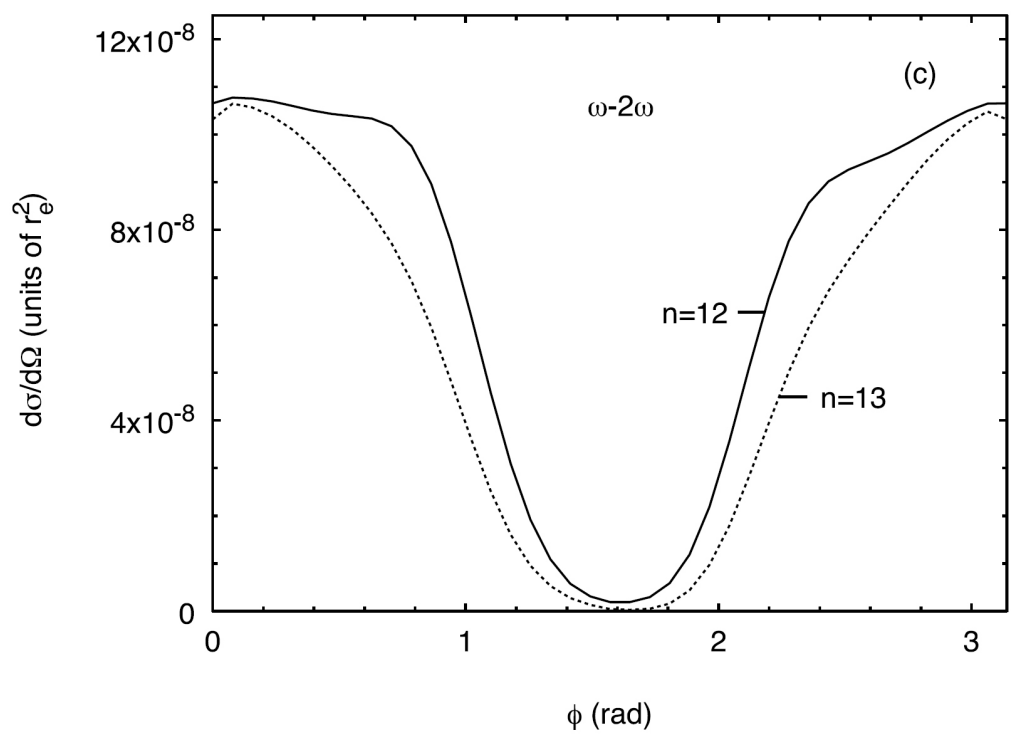

Figure 4. (continued)

The first term on the right-hand side of Equation (14) is just the classical kinetic energy of the electron, which is born with the momentum $\pm p_{0} \hat{e}$ in the laser field $A(t) \hat{e}$ at time $t_{0}$ and which has been propagated in the laser field until time $t_{1}$. The condition $\partial(n \omega)=\partial t_{0}$ gives

$$
\left[-E\left(t_{1}\right) \frac{\partial t_{1}}{\partial t_{0}}+E\left(t_{0}\right)\right]\left[A\left(t_{1}\right)-A\left(t_{0}\right) \pm p_{0}\right]=0 .
$$

The term $\partial t_{1}=\partial t_{0}$ can be obtained by taking the derivative over $t_{0}$ of the last equality in Equation (11). It follows that

$$
\left[A\left(t_{1}\right)-A\left(t_{0}\right) \pm p_{0}\right] \frac{\partial t_{1}}{\partial t_{0}}= \pm p_{0}-\left(t_{1}-t_{0}\right) E\left(t_{0}\right) .
$$

From Equations (15) and (16) we obtain finally the condition

$$
\boldsymbol{E}\left(t_{0}\right)\left[\boldsymbol{A}\left(t_{1}\right)-\boldsymbol{A}\left(t_{0}\right)+\left(t_{1}-t_{0}\right) \boldsymbol{E}\left(t_{1}\right)\right]= \pm p_{0}\left[\boldsymbol{E}\left(t_{1}\right)-\boldsymbol{E}\left(t_{0}\right)\right]
$$

We solve the system of equations given by Equation (17) (which gives the extremum condition for $n \omega$ ) and the second equality of Equation (11) (which specifies the return of the electron to the nucleus) for $t_{0}$ and $t_{1}$ with the additional condition that $t_{1}>t_{0}$, i.e., that the return time $\tau>0$. We find the extrema of $n \omega$ by introducing the resulting values for $t_{0}$ and $t_{1}$ into Equation (14). There are two solutions for $n \omega$, one for $+p_{0}$ and the other for $-p_{0}$. In Figures $5(\mathrm{a})$ and (b) we present these solutions as functions of the relative phase $\phi$ for the $(\omega, 3 \omega)$ and $(\omega, 2 \omega)$ cases, respectively. In the $(\omega, 3 \omega)$ case the solutions for $+p_{0}$ and $-p_{0}$ coincide. This property follows from the fact that (for $r=3)(E, A, \alpha) \rightarrow(-E,-A,-\alpha)$ for $t \rightarrow t+\pi /$ $\omega$, so that Equations (11), (14), and (17) are invariant to the simultaneous substitution $t \rightarrow t+\pi / \omega$ and $+p_{0} \rightarrow-p_{0}$. Analogously, in the $(\omega, 2 \omega)$ case we have $(\boldsymbol{E}, \boldsymbol{A}$, $\alpha) \rightarrow(-E,-A,-\alpha)$ for $t \rightarrow t+\pi / \omega$ and $\phi \rightarrow \phi+\pi$, so that in this case we have the symmetry $\left(p_{0}, \phi\right) \leftrightarrow\left(-p_{0}, \phi+\pi\right)$. This is consistent with the $\phi \leftrightarrow \phi+\pi$ symmetry of the DCS for $r=2$. In the $(\omega, 3 \omega)$ case the minimum value of $n \omega$ ranges between -31.1 and -23.0 as $\phi$ varies, while the maximum ranges from -29.8 to 3.1. This explains why in Figure 1 the cutoff for negative values of $n$ is around -30 , while for positive $n$ we do not have a clear plateau. In the $(\omega, 2 \omega)$ case the solutions for 

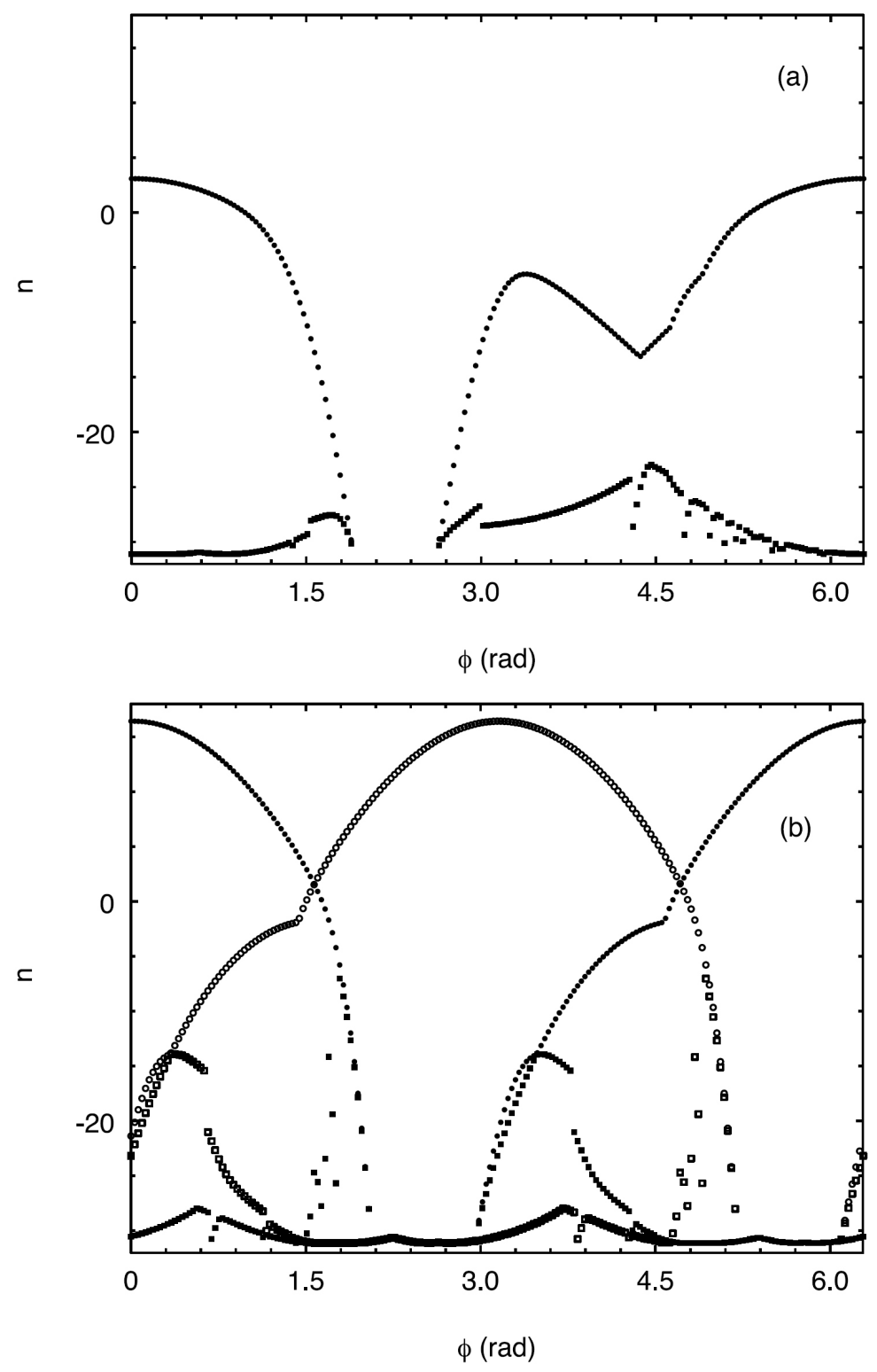

Figure 5. Extremal values of the number $n$, obtained using the quasi-classical method presented in section 5 , as functions of the relative phase for the bichromatic laser field of frequencies (a) $(\omega, 3 \omega)$ and $(\mathbf{b})(\omega, 2 \omega)$. The solutions obtained for $+p_{0}$ (see the text for definition) are represented by the full symbols, while the solutions for $-p_{0}$ are represented by the open symbols. Note, however, that in (a) the solutions for $\pm p_{0}$ coincide. The squares (circles) correspond to the minimum (maximum) values of $n$.

$+p_{0}$ and $-p_{0}$ are complementary in the sense that for $0 \leq \phi \leq \pi / 2$ and $3 \pi / 2 \leq \phi \leq$ $2 \pi$ the extrema of $n \omega$ are determined by the solutions for $+p_{0}$, while for $\pi / 2 \leq \phi$ $\leq 3 \pi / 2$ the extrema are determined by the solutions for $-p_{0}$. Therefore, the minimum value of $n$ is always around -31 , which is in agreement with the results presented in Figure 3. For positive values of $n$, the maxima are at 16.4 for $\phi=0, \pi$, 
which agrees with the position of the plateau and the cutoff in Figure 3(a). The smallest maximum value of $n$ is 1.62 for $\phi=\pi / 2$, which explains the absence of a clear plateau for $n>0$ in Figure 3(b).

\section{Conclusions}

We have, in this paper, extended the results of the paper by Miloševíc and Ehlotzky (1998) to the case of x-ray-atom scattering in the presence of a bichromatic laser field. It has been shown that the DCS in the $(\omega, 2 \omega)$ case possesses the symmetry $\mathrm{d} \sigma(\phi) / \mathrm{d} \Omega=\mathrm{d} \sigma(\phi+\pi) / \mathrm{d} \Omega$. It has also been shown that, according to parity conservation, the energy $n \omega$ exchanged with the laser field is such that the number $n$ is even for the $(\omega, 3 \omega)$ case, while it can be either even or odd for the $(\omega, 2 \omega)$ case. The numerical results presented show that the bichromatic DCS increases by many orders of magnitude compared with the monochromatic one. The results obtained strongly depend on the relative phase $\phi$, so that coherent phase control of the process is shown to be possible. For some values of $\phi$ the plateau for positive values of $n$ becomes significant, which indicates that it is possible to increase the energy of the scattered x-ray photons. We have also presented a quasi-classical explanation of this effect.

\section{Acknowledgments}

This work has been supported in part by the National Science Foundation under grant number PHY9722110.

\section{References}

Chang, Z., Rundquist, A., Wang, H., Murnane, M. M., and Kapteyn, H. C. 1997 Phys. Rev. Lett. 79: 2967 1999 Phys. Rev. Lett. 82: 2006 (erratum)

Glover, T. E., Schoenlein, R. W., Chin, A. H., and Shank, C. V. 1996 Phys. Rev. Lett. 76: 2468

Ilkov, F. A., Decker, J. E., and Chin, S. L. 1992 J. Phys. B: At. Mol. Opt. Phys. 25: 4005

Lambropoulos, P., and Walther, H. (ed) 1997 Multiphoton Processes 1996 (Institute of Physics Conference Series 154) (Bristol: IOP)

Lewenstein, M., Balcou, Ph., Ivanov, M. Yu., L'Huillier, A., and Corkum, P. B. 1994 Phys. Rev. A 49: 2117

Miloševíc, D. B., and Ehlotzky, F. 1997 Phys. Rev. A 56: 3879 1998 Phys. Rev. A 58: 2319

Miloševíc, D. B., and Piraux, B. 1996 Phys. Rev. A 54: 1522

Miloševíc, D. B., and Starace, A. F. 1998 Phys. Rev. Lett. 81: 5097

Muller, H. G., and Fedorov, M. V. (eds) 1996 Super-Intense Laser-Atom Physics IV (Dordrecht: Kluwer)

Schins, J. M., Breger, P., Agostini, P., Constantinescu, R. C., Muller, H. G., Grillon, G., Antonetti, A., and Mysyrowicz, A. 1994 Phys. Rev. Lett. 73: 2180

Schnürer, M., et al. 1998 Phys. Rev. Lett. 80: 3236

Spielmann, C., Burnett, N. H., Sartania, S., Koppitsch, R., Schnürer, M., Kan, C., Lenzner, M., Wobrauschek, P., and Krausz, F. 1997 Science 278: 661 